\title{
Ser enfermeira intensivista: elementos identitários presentes no campo das representações sociais
}

\author{
Being an intensive care nurse: identity elements present in the field of social representations \\ Ser enfermera intensivista: elementos de identidad presentes en el campo de las representaciones sociales
}

Rafael Celestino da Silva', Márcia de Assunção Ferreira", Thémis Apostolidis"I

\begin{abstract}
RESUMO
Objetivo: identificar os aspectos identitários presentes nas representações sociais de enfermeiras sobre as práticas de cuidar do cliente hospitalizado na terapia intensiva. Método: estudo de campo, qualitativo, descritivo-explicativo, apoiado na teoria das representações sociais e no conceito de identidade social. Após aprovação do projeto pelo Comitê de Ética em Pesquisa, realizaram-se entrevistas com 21 enfermeiros atuantes na assistência de uma unidade de terapia intensiva de um hospital federal, com uso de um roteiro semiestruturado de questões, cujos dados foram submetidos à análise de conteúdo temática. Resultados: evidenciam-se aspectos de identificação social e valorização do endogrupo, e de comparação social e estereotipização do exogrupo, quais sejam os modos de usar as tecnologias, o delineamento da figura típica do paciente, bem como os atributos do enfermeiro ideal, o sentido do trabalho, o relacionamento intra e interequipes. Conclusão: recomenda-se reorganizar o campo da representação social a partir de intervenções sob elementos da identidade.
\end{abstract}

Descritores: Cuidados de enfermagem; unidades de terapia intensiva; identidade social; psicologia social.

\section{ABSTRACT}

Objective: to recognize the identity aspects present in the social representation of nurses about the care practices for the client hospitalized in intensive therapy. Method: field study, qualitative, descriptive and explanatory, based on the theory of social representations and on the social identity concept. After approval by the Research Ethics Committee, interviews were carried out with 21 nurses working in the assistance of an intensive care unit in a federal hospital, using a semi-structured guide. Data were submitted to the content analysis. Results: social identification and enhancement of the group itself, and social comparison and stereotyping of the outside group are evidenced, which are: the ways of using the technologies, the delineation of the typical patient figure, as well as the attributes of the ideal nurse, the sense of work, the intra and intercompany relationship. Conclusion: it is recommended the reorganization of the field of social representation from interventions under identity elements. Descriptors: Nurse care; intensive therapy unit; social identity; social psychology.

\section{RESUMEN}

Objetivo: reconocer los aspectos de identidad presentes en la representación social de las enfermeras sobre las prácticas de cuidado para el cliente hospitalizado en terapia intensiva. Método: estudio de campo, cualitativo, descriptivo y explicativo, basado en la teoría de las representaciones sociales y en el concepto de identidad social. Después de la aprobación del Comité de Ética en Investigación, se realizaron entrevistas con 21 enfermeras que trabajaban en la asistencia de una unidad de cuidados intensivos en un hospital federal, utilizando una guía semiestructurada. Los datos fueron sometidos al análisis de contenido. Resultados: se evidencia la identificación social y la mejora del grupo en sí, y la comparación social y los estereotipos del grupo externo, que son: las formas de usar las tecnologías, la delineación de la figura típica del paciente, así como los atributos de la enfermera ideal, el sentido del trabajo, la relación intra e intercompañía. Conclusión: se recomienda la reorganización del campo de la representación social a partir de intervenciones bajo elementos de identidad.

Descriptores: Atención en enfermería; unidades de terapia intensiva; identidad social; psicología social.

\section{INTRODUÇÃO}

No âmbito da teoria das representações sociais parte-se da premissa de que a conformação do pensamento humano se dá com base nas várias inserções grupais dos atores, tais como no campo social, histórico, político, cultural, dentre outros ${ }^{1}$. Esta inscrição de membros individuais em um espaço social produz o sentimento de pertença, a partir do qual se define a identidade. Assim, reconhece-se que as representações sociais (RS), por meio das práticas, trazem à tona a questão da identidade social, que expressa uma po- sição e uma maneira de ser no mundo² e faz parte do contexto do saber.

A discussão deste tema da identidade perpassa várias disciplinas. No campo da educação, por exemplo, há interesses sobre os modos de construção da identidade profissional dos docentes da educação básica, considerando as representações sociais da profissão. Tais modos se relacionam com as estruturas culturais e sociais historicamente determinadas, a partir das quais os professores reconstroem sua identidade ${ }^{3}$.

'Doutor em Enfermagem. Professor Adjunto do Departamento de Enfermagem Fundamental da Universidade Federal do Rio de Janeiro. Brasil. Email: rafaenfer@yahoo.com.br. "Doutora em Enfermagem. Professora Titular do Departamento de Enfermagem Fundamental da Universidade Federal do Rio de Janeiro. Brasil. Email: marcia.eean@gmail.com. "'Doutor em Psicologia. Diretor do Laboratório de Psicologia Social da Aix-Marseille Université. France. E-mail: themistoklis.apostolidis@univ-amu.fr. 
Já na Saúde Coletiva, a preocupação se articula com a atuação profissional e identidade do "novo sanitarista", em face da criação dos cursos de graduação em Saúde Coletiva. Ao investigar as percepções de estudantes e egressos destes cursos de graduação concluiu-se que embora a maioria não tivesse se interessado inicialmente por essa área, com as experiências do curso vão adquirindo pertencimento, o qual se relaciona com a identificação com elementos políticos, sociais e valores da prática nesse campo ${ }^{4}$.

$\mathrm{Na}$ enfermagem, disciplina de interesse do artigo em tela, a produção de conhecimento acerca deste tema se assenta na análise da constituição da identidade profissional. Em estudo que fez uma análise filosófica desta identidade, afirma-se que sua origem se deu a partir das proposições nightingaleanas em 1860, quando se demarca o que é e o que não é enfermagem ${ }^{5}$. Tal identidade profissional vem se conformando ao longo da construção sócio-histórica da profissão, como aponta pesquisa com egressos de um curso de graduação em enfermagem da década de 1970 sobre o sentido e significado de ser enfermeiro no momento da opção profissional. A identidade destes profissionais abrangeu: a enfermagem como segunda opção; o cuidado como sinônimo de fazer; o estigma social da profissão; a possibilidade de inserção no mercado de trabalho e de ascensão social ${ }^{6}$.

Em algumas áreas de atuação da enfermagem, como é o caso das especializadas, a discussão em torno da existência de uma identidade profissional da enfermeira também se faz presente, no sentido de compreender e explicar seu comportamento e atuação nestes cenários assistenciais, como é o caso das unidades de terapia intensiva (UTI).

Alguns autores defendem que neste ambiente há uma clara identidade profissional, de caráter singular e que abarca todos os agentes de saúde implicados nele. Quando se escolhe trabalhar na UTI delineia-se por contraste a outros setores do hospital uma identidade específica e comum a diversas categorias profissionais: a do ser intensivista7, a partir da qual se reivindicam competência específica, compartilhamento de valores comuns e exercício do controle sobre quem lá pode atuar. Os enfermeiros se unem por um sentimento de pertencimento, a partir do qual dão sentido ao mundo, lidam praticamente com o cotidiano e estabelecem relações comunicativas.

A falta de compreensão desta identidade social fomenta a criação de estereótipos sobre a assistência de enfermagem na UTI, cerne da problemática desta pesquisa. Estes estereótipos se organizam a partir dos questionamentos, notáveis na literatura, acerca da maneira de se comportar dos profissionais intensivistas, marcada por momentos em que o paciente é visto como objeto, despersonalizando as relações de cuidado, e preocupando-se com o fazer técnico de alta complexidade ${ }^{8,9}$ bem como sobre as implicações de suas ações à segurança ${ }^{10-12}$.
Neste sentido, considerando a necessidade de aprofundamento sobre os elementos que influenciam nos modos de agir profissional, com vistas à adoção de estratégias voltadas a intervenção sobre estes elementos, de modo a favorecer a qualidade assistencial, justifica-se o desenvolvimento desta pesquisa. Em estudo prévio sobre as RS de enfermeiros atuantes em UTI sobre suas práticas verificou-se que estas representações se organizam em torno da tecnologia e do paciente de UTI, dando origem a estilos de cuidar próprios deste cenário ${ }^{13}$.

Então, questiona-se: que aspectos identitários existem nas representações sociais das práticas de cuidar do cliente hospitalizado na terapia intensiva de enfermeiras atuantes neste cenário? Objetiva-se identificar os aspectos identitários presentes nas RS das práticas de cuidar do cliente hospitalizado na terapia intensiva de enfermeiras atuantes neste cenário.

\section{RefERENCIAL TEÓRICO}

O estudo apoiou-se na teoria das representações sociais e no conceito de identidade social. Na perspectiva da psicologia social desenvolvida por Deschamps e Moliner a identidade pode ser concebida como um fenômeno subjetivo e dinâmico resultante da dupla constatação de similitude e de diferença entre si, o outro e outros grupos ${ }^{14}$. As RS concorrem para tal identidade comum aos indivíduos, e esta, por sua vez, fomenta as identificações e diferenciações sociais com base na afiliação e pertencimento a um grupo social. Assim, a categorização social entre quem somos e quem não somos permite compreender e ordenar a realidade, determinando a identidade ${ }^{15}$.

Sob esta ótica os referenciais escolhidos justificam-se, pois serão aplicados na tentativa de apreender como se dão os processos de categorização e comparação social, a partir dos quais se define a identidade e as práticas empreendidas pelo grupo dos intensivistas, estabelecendo com base nisso interfaces com as RS elaboradas por estes enfermeiros sobre sua prática.

\section{Metodologia}

Estudo de campo, de abordagem qualitativa e cunho descritivo-explicativo, com aplicação da vertente processual da teoria das representações sociais. O campo foi um hospital federal do município do Rio de Janeiro, e o lócus a UTI desta instituição. Do universo de 24 enfermeiros que trabalhavam nesta UTI participaram 21, que atuavam diretamente na assistência ao cliente e que estavam presentes durante o período destinado à pesquisa. Os três restantes foram excluídos por estarem de férias ou licença.

Os dados foram produzidos por meio de entrevista individual. As entrevistas foram realizadas no próprio setor, no período da tarde, tendo uma duração média de 1 hora e 30 minutos. Utilizou-se um roteiro de coleta 
de dados sociodemográficos a fim de caracterizar os participantes e um roteiro de entrevista semiestruturada voltado a explorar o objeto, com questões que abarcavam sua prática diária na UTI, estilo de cuidar, emprego de tecnologias dentre outros.

Os dados oriundos das entrevistas passaram por análise de conteúdo temática, a partir da realização das seguintes etapas: identificação dos temas mais frequentes e significantes, organização dos núcleos de sentido, categorização, possibilitando definir dois eixos de análise. O estudo foi aprovado pelo Comitê de Ética em Pesquisa da instituição que serviu de lócus para investigação, sob número de parecer: 35/10. O anonimato dos participantes foi respeitado mediante sua identificação por meio do código Enf (enfermeiro) , seguido de número arábico conforme a ordem de captação dos participantes. A produção dos dados ocorreu de janeiro a junho de 2011.

\section{RESUltAdos E Discussão}

\section{Nós: elemento de pertença dos enfermeiros intensivistas}

As identidades sociais são compartilhadas por aqueles que ocupam as mesmas posições, que têm pertenças em comum e similitudes. Tal identificação social presente nos dados em 17 unidades de registro (UR) é demarcada quando os depoentes se reportam à sua prática de cuidar, que caracteriza ser enfermeiro intensivista, qual seja: um profissional que busca estratégias para proporcionar uma atenção de qualidade ao cliente, incorporando-se a ela o uso de tecnologias e o aprimoramento constante do conhecimento que fundamente sua utilização.

Eles têm uma visão de ser prático por conta da tecnologia, por conta do cuidado, a gente tem como fazer vários procedimentos em um paciente só. Não tem uma visão muito diferente da minha não, enquanto intensivista é mais ou menos isso, a questão do intensivista de tentar resolver, tem aquele paciente grave, monitorização constante da máquina. (Enf. 4)

Um dos elementos que sustenta a similitude intragrupo e estimula a pertença é o uso das tecnologias no cuidado, verificada em 14 UR temática. Nesta direção, as enfermeiras conferem grande importância a este componente de sua identidade, na qual se utilizar das tecnologias dando-Ihe o valor apropriado significa estar em consonância com a prática esperada do intensivista.

A gente que trabalha em UTI, se não tiver nenhum monitor aqui, não precisa colocar paciente aqui, não precisa chamar UTI [...] é uma enfermaria, uma clínica $e$ eu vou ter o cuidado de verificar os sinais vitais de duas em duas horas [...] faz parte de nossa vida dentro da UTI, faz parte da equipe, é todo um envolvimento mesmo, não tem como fugir mais, já era [...]. (Enf. 4)

O componente emocional desta pertença dirigido para o próprio grupo vem à tona quando se alcança a recuperação do cliente hospitalizado na UTI. Emerge então a valoração positiva de fazer parte deste grupo e atuar em tal cenário, demonstrando-se satisfação com o trabalho desenvolvido e alto nível de comprometimento. Esta temática esteve presente em 09 UR.

Você lida com essas situações de dor, de sofrimento, e é bom depois você ver o paciente sair, ver o doente indo lá, agradecendo a gente [...] é gratificante você ver que todo aquele seu esforço, aquela noite toda que você passou ali, o menino ficou bem, sobreviveu. Mas isso é a UTI do dia-a-dia. (Enf. 20 )

Este sentimento positivo dá sentido à maior preferência destes profissionais pelos clientes por eles classificados como mais graves, como forma de proteção do endogrupo e fortalecimento da afiliação de seus membros. Isto porque a partir desta escolha torna-se mais visível a participação da enfermeira na recuperação do cliente, quando comparado a um cliente acordado e menos grave.

Quem trabalha com terapia intensiva gosta de ver o paciente grave porque a gente gosta de trabalhar, mas assim a satisfação é imensa quando você vê um paciente gravíssimo ir melhorando, melhorando [...] Eu gosto de pegar o paciente grave e vê-lo acordar, ser extubado e sair daqui falando. (Enf. 18)

\section{Eles: entre diferenças e estereótipos}

A similitude traz consequências importantes na percepção dos grupos. Isto porque quanto mais o indivíduo teria identificação a um grupo, mais ele teria diferenciação deste grupo em relação a outros. Então, o efeito do contraste se manifesta por uma exacerbação das diferenças entre as categorias, na qual existe uma valorização do endogrupo e uma desvalorização do exogrupo, atribuindo-lhe traços comuns - estereótipos. A valorização é percebida ao tratarem do reconhecimento do enfermeiro de UTI, atribuindo-o o status de profissional com maior conhecimento em 12 UR, e delineando requisitos considerados imprescindíveis ao cuidado de enfermagem na UTI para quem lá vai atuar com 14 UR.

Mas o enfermeiro de terapia intensiva é sempre visto como o enfermeiro que detêm maior conhecimento. (Enf. 13)

Conhecimento técnico-científico, porque você está lidando com um paciente com um grau de complexidade maior que de outro setor e com tecnologia. (Enf.2)

Como consequência emerge a avaliação negativa das enfermeiras que atuam em setores de clínica, que é estereotipada como aquela de menor saber, inferior ao da enfermeira intensivista.

Eles acham um bicho de sete cabeças. Acha que é um mundo totalmente, eu posso dizer por que fui remanejada para a clínica. Aí eu fiquei uns 03 dias na clínica. [....] Realmente as pessoas não sabem nada. Acho que todas as pessoas têm que passar na UTI, porque a UTI é uma escola. (Enf.1)

A demarcação dos espaços de dentro e fora da terapia intensiva é outro exemplo que ajuda a ilustrar a desvalorização do exogrupo. Isto é notável quando a comparação é feita em relação aos outros mem- 
bros da equipe multiprofissional que não trabalham exclusivamente neste setor, e constituem a estrutura externa à UTI.

No geral, o relacionamento é bom, a maioria é pessoas que estão ali, a maior parte do tempo, os fisioterapeutas, médicos e enfermeiros. Com relação às pessoas de fora, da terapia nutricional, a gente não tem uma relação, fala o básico, eles falam às vezes coisas que a gente não concorda. (Enf. 17)

A dialética entre o endogrupo e o exogrupo, nós versus eles termina por ser retratada na discussão acerca da imagem da enfermeira de terapia intensiva, que obteve 16 UR. Observa-se abaixo entre as intensivistas (endogrupo) uma autoimagem positiva e, entre as demais enfermeiras, uma tendência de avaliação negativa das intensivistas (exogrupo).

Acham que somos uns nojentos, metidos [...] O que acontece é uma classificação que a gente tem mais habilidade com as tecnologias do que os outros [...] numa situação de emergência, as pessoas mais preparadas para lidar com essas situações, são as que têm mais domínio da tecnologia [...] em alguns casos pode ser de admiração, vê que a pessoa domina uma determinada situação [...]. (Enf. 7)

Esta questão da identidade vem sendo considerada pelos estudiosos no âmbito das RS como elemento central desta teoria, mormente na organização do campo da RS, já que o indivíduo projeta a identidade social no objeto que representa ${ }^{16}$. Um estudo clássico no qual a discussão da identidade ganha evidência é o das representações transculturais da AIDS ${ }^{17}$.

Neste, a autora mostra que um sentimento de defesa dá sustentação à formação das RS da AIDS, tendo como base a seguinte lógica: Eu não, o meu grupo não, ou seja, os sujeitos buscam desviar a atenção da ameaça trazida pela AIDS ao Eu (grupo interno), deslocando o seu olhar para o outro, para quem passa a ser projetada a responsabilidade por sua origem e desenvolvimento (grupo externo - ameaçado e ameaçador) ${ }^{17}$.

Mais recentemente outros grupos têm recebido atenção pelos pesquisadores no que tange a estas relações intergrupais, como é o caso dos ciganos e rurais. Neste sentido, buscou-se analisar as relações intergrupais que se delineavam entre mulheres ciganas e rurais, e conhecer as representações e práticas construídas nas relações endo e exogrupais ${ }^{15}$.

A representação do exogrupo cigano é pautada por um sentimento de medo e percepção dos ciganos como amaldiçoados. A representação do exogrupo rural entre os ciganos é pouco clara, mas reforça uma tendência de criação de autoimagem positiva, como forma de valorização do seu grupo de pertença em comparação aos outros. No que concerne às representações do endogrupo, observa-se a ascendência de elementos positivos na construção da identidade social de cada uma delas ${ }^{15}$.

Este exemplo ilustra bem o papel que a identidade desempenha no nosso cotidiano e de como esta interfere nas relações sociais com o outro. Tais relações são orientadas pelos processos de identificação e diferenciação que, por sua vez, são mediadores dos significados atribuídos a si e aos outros, atestando a imbricação entre RS e identidade ${ }^{18}$.

No caso da temática deste estudo, outros resultados de pesquisa trazem subsídios à discussão na interface com os dados apresentados nesta investigação. Um deles foi produzido a partir de uma etnografia em uma UTI de um hospital público universitário ${ }^{7}$. Dentre os eixos de análise consta o intensivista como identidade profissional, no qual um conjunto de elementos sustenta a construção de tal identidade.

No topo do hospital é um desses elementos, ou seja, a pesquisadora percebeu que os participantes expressavam grande satisfação em trabalhar em um serviço: tido como de excelência, numa posição hierárquica superior a outros serviços do hospital, com profissionais de alto padrão, maior organização da dinâmica do serviço e equipe multidisciplinar. Além disso, havia uma autoimagem de heroísmo, de salvador, que alimentava um sentimento de gratificação quando se alcançava a recuperação do cliente ${ }^{7}$.

Tornar-se intensivista/os bambambãs é outro constituinte da identidade deste profissional. O super é usado para designá-lo, sempre acima de seus pares, os quais são vistos como de menor capacitação ou até ignorantes. Sua autoimagem é de superioridade aos seus colegas, ancorado no conhecimento que possui e domínio da tecnologia que maneja ${ }^{7}$. Assim, embora discordem da imagem que os colegas têm deles de elite/ bambambãs, que a distancia desses pares, sentem-se orgulhosos de sua função?.

O pertencimento grupal dos intensivistas é reforçado por mais estudiosos, que mostram que a criação de uma identidade com a UTI pelos enfermeiros tem relação com os elementos que produzem o sentimento de prazer nestes profissionais, quais sejam: o fato de ajudar na recuperação do paciente grave, manejar os equipamentos, buscar continuamente aperfeiçoamento, ser reconhecido pelo trabalho desenvolvido ${ }^{19}$. Esse aspecto da satisfação e do prazer no trabalho é reforçado no estudo que buscou conhecer a perspectiva de médicos sobre a saúde e o trabalho na UTI neonatal de um hospital público. Nas entrevistas realizadas sobressai o gosto por trabalhar na UTI, principalmente pela ideia de maior controle sobre as intercorrências no quadro das crianças internadas a partir do uso dos equipamentos ${ }^{20}$.

Acerca do conhecimento superlativizado da enfermeira intensivista, veiculado como uma das suas bases identitárias, este foi entendido como a essência da estrutura representacional do cuidado intensivo para profissional de saúde de UTI móvel ${ }^{21}$. Por fim, a dimensão tecnológica das práticas de cuidado da enfermagem na UTI tem assento numa série de pesquisas 
nacionais e internacionais, que valorizam a necessidade do intensivista conferir atenção a essas tecnologias no dia-a-dia ${ }^{22-24}$ como forma de manter a vida dos clientes, e, ao mesmo tempo, expressar o seu conhecimento, o que duplamente reforça sua identidade profissional e justifica a primazia das tecnologias na terapia intensiva.

Um exemplo da necessidade de atenção às tecnologias pode ser visualizado na pesquisa que identificou as falhas dos equipamentos durante o seu manejo pelos enfermeiros, a qual evidenciou problemas com o funcionamento das bombas infusoras e com as baterias dos ventiladores artificiais, além dos erros dos profissionais ligados ao desenho do equipamento. Esses resultados ressaltam a importância de que o enfermeiro realize a adequada gestão dos equipamentos na UTI, a tecnovigilância, de modo a prevenir a ocorrência de incidentes que põem em risco a segurança dos pacientes ${ }^{24}$.

Tendo em vista os dados e o entendimento do conceito de identidade social evidencia-se que estas representações são atravessadas por aspectos de cunho identitário, de um lado os de identificação social e de valorização do endogrupo, quais sejam: a postura vigilante e interventiva do intensivista, o emprego de tecnologias/máquinas no cuidado, o sentimento positivo de recuperar um cliente grave escolhido para cuidar, o reconhecimento profissional com base no conhecimento; do outro os de comparação social, que estereotipa e desvaloriza o grupo de enfermeiras que não atuam na terapia intensiva e vice-versa.

Neste caso, o perfil estabelecido de enfermeiro de terapia intensiva alicerçado no saber/conhecimento leva a uma avaliação negativa dos outros profissionais, considerados de fora, sendo este o ponto de partida para os conflitos entre as equipes. Por conseguinte, isto gera também o movimento contrário de depreciação das enfermeiras intensivistas vistas como mecânicas, tecnológicas, desumanas.

Essa questão da humanização do cuidado na terapia intensiva é, inclusive, preocupação marcante na literatura da área, sendo objeto de diferentes análises ${ }^{9,25,26}$. Uma das análises identificada é sobre as relações dos profissionais de enfermagem com os pacientes e familiares de uma UTI à luz das concepções de Paterson e Zderad ${ }^{25}$.

Os autores afirmam que há uma relação de poder dos profissionais com os pacientes e distanciamento dos familiares, sem estabelecimento de uma relação dialógica que valorize o encontro e a subjetividade. Logo, concluem da necessidade de humanizar tal assistência, possibilitando a expressão das dimensões objetivas e subjetivas dos pacientes e familiares ${ }^{25}$. Essa valorização da dimensão subjetiva, que fortalece o compromisso e a responsabilização, também é realçada em segunda investigação como um dos elementos capaz de promover a humanização da gestão do cuidado de enfermagem na UTI ${ }^{26}$.
Portanto, a partir de tal identidade, constroem-se imagens classificadoras dos profissionais, estabelecendo-se métricas de intensidade que diferenciam os profissionais como mais capazes/menos capazes, demarcando claramente a posição de cada grupo e de suas práticas, o que influencia na relação entre elas.

\section{CONCLUSÃo}

Os componentes identitários apreendidos no estudo se articulam no campo das RS a outros elementos de ordem normativa, afetiva e simbólica e contribuem para os sentidos atribuídos por estes profissionais à sua prática de cuidar na terapia intensiva. Afirma-se então que estas RS estão revestidas pela identidade social da enfermeira intensivista, que abarca elementos orientadores dos modos de usar as tecnologias, o delineamento da figura típica de paciente da UTI bem como os atributos do enfermeiro ideal, o sentido do trabalho, o relacionamento intra e interequipes. Este conjunto de fatores se liga de modo a configurar as práticas de cuidar das enfermeiras, dando, por fim, um significado particular a categoria social de ser intensivista.

Reconhece-se inicialmente limitações teóricas pelos resultados estarem associados ao contexto de produção, porém, aponta possibilidades para o estudo da identidade em novos cenários e com mais sujeitos, ampliando o poder de generalização. Levando-se em conta tal afirmação, a categoria Ser enfermeiro intensivista revela aspectos que podem ser foco de intervenções que promovam a aplicação prática dos resultados da pesquisa.

Nesta ótica, entendendo o campo da RS como um conjunto estruturado de significados, saberes e informações e sendo a identidade um dos aspectos que o integra, este pode ser reorganizado a partir de grupos reflexivos que permitam a interferência sob alguns elementos da identidade que ganham destaque a partir da análise dos dados: nas tecnologias, quando supervalorizadas na gerência à distância do cuidado do cliente; na tipificação do paciente de UTI, que por consequência subestima o menos grave, mais acordado e suas queixas subjetivas; o superlativo do enfermeiro de UTI, que inferioriza o saber do outro e é motivo de conflitos.

\section{REFERÊNCIAS}

1. Rocha LF. Teoria das representações sociais: a ruptura de paradigmas das correntes clássicas das teorias psicológicas. Psicol. cienc. prof. 2014 [citado em 10 jan 2018]; 34(1):46-65. DOI: http:// dx.doi.org/10.1590/S1414-98932014000100005

2. Jodelet D. A representação: noção transversal, ferramenta da transdisciplinaridade. Cad. Pesqui. 2016 [cited 2018 Jan 10]; 46(162): 1258-71. DOI: http://dx.doi.org/10.1590/198053143845. 3. Gebran RA, Trevizan Z. Social representations in the construction of professional identity and teachers' activity. Acta Sci. Educ. 2018 [cited 2018 Jan 10]; 40(2): e34534. DOI: http://dx.doi. org/10.4025/actascieduc.v40i2.34534

4. Silva VO, Pinto ICM. Identidade do sanitarista no Brasil: percepções de estudantes e egressos de Cursos de Graduação em Saúde Pública/Coletiva. Interface (Botucatu). 2018 [citado em 10 
jan 2018]; 22(65): 539-50. DOI: http://dx.doi.org/10.1590/180757622016.0825

5. Carvalho V. Sobre a identidade profissional na Enfermagem: reconsiderações pontuais em visão filosófica. Rev. bras. enferm. (Online). 2013 [citado em 10 jan 2018]; 66(esp): 24-32. DOI: http:// dx.doi.org/10.1590/S0034-71672013000700003

6. Teodosio SS, Padilha, MI. To be a nurse: a professional choice and the construction of identity processes in the 1970s. Rev. bras. enferm. (Online). 2016 [cited 2018 Jan 18]; 69(3): 401-7. DOI: http://dx.doi.org/10.1590/0034-7167.2016690303i

7. Menezes RA. Difíceis decisões: etnografia de um centro de tratamento intensivo. Rio de Janeiro: Editora Fiocruz; 2006

8. Reis CCA, Sena ELS, Fernandes MH. Humanization care in intensive care units: integrative review. Rev. pesqui. cuid. fundam. (Online). 2016 cited 2018 Jan 10]; 8(2): 4212-22. DOI: http:// dx.doi.org/10.9789/2175-5361.2016.v8i2.4212-4222

9. Nascimento ERP, Gulini JEHMB, Minuzzi AP, Rasia MA, Danczuk RFT, Souza BC. As relações da enfermagem na unidade de terapia intensiva no olhar de Paterson e Zderad. Rev. enferm. UERJ. 2016 [citado em 10 jan 2018]; 24(2): e5817. DOI: http://dx.doi. org/10.12957/reuerj.2016.5817

10. Abraham J, Kannampallil T, Brenner C, Lopez KD, Almoosa KF, Patel $B$, et al. Characterizing the structure and content of nurse handoffs: a sequential conversational analysis approach. J. Biomed. Inform. 2016 [cited 2018 Jan 10]; 59: 76-88. DOI: http:// dx.doi.org/10.1016/j.jbi.2015.11.009

11. Oliveira JGAD, Almeida LF, Hirabae LFA, Andrade KBS, Sá CMS, Paula VG. Interruptions in intensive care nursing shift handovers: patient safety implications. Rev. enferm. UERJ. 2018 [cited 2018 Dec 10]; 26: e33877. DOI: https://dx.doi.org/10.12957/ reuerj.2018.33877

12. Mendes RNC, Carmo AFS, Haddad MCL, Rossaneis MA. Percepções de enfermeiros sobre o uso de equipamentos na Unidade de Terapia Intensiva. Rev. enferm. UFPE on line. 2014 [citado em 10 Jan 2018]; 8(7): 1904-11. DOI: https://dx.doi.org/10.5205/ reuol.5963-51246-1-RV.0807201411

13. Silva RC, Ferreira MA, Apostolidis T. Estilos de cuidar de enfermeiras na Terapia Intensiva mediados pela tecnologia. Rev. bras. enferm. (Online). 2014 [citado em 12 jan 2018]; 67(2): 252-60. DOI: http://dx.doi.org/10.5935/0034-7167.20140034

14. Deschamps JC, Moliner P. L'identité en psychologie sociale: des processus identitaires aux représentations sociales. Paris $(\mathrm{Fr})$ : Armand Colin; 2011.

15. Bonomo M, Trindade ZA, Souza L, Coutinho SMS. Representações sociais e identidade em grupos de mulheres ciganas e rurais. Psicologia [Internet]. 2008 [citado em 29 mai 2017]; 22(1): 153-81. Disponível em: www.scielo.mec.pt/pdf/psi/v22n1/v22n1a07.pdf
16. Seidmann S. Identidad personal y subjetividad social: educación y constitución subjetiva. Cad. Pesqui. 2015 [cited 10 jan 2018] 45(156): 344-57. DOI: http://dx.doi.org/10.1590/198053143204 17. Joffe H. "Eu não, "o meu grupo não": Representações transculturais da AIDS. In: Jovchelovitch S, Guareschi P, organizadores. Textos em representações sociais. Petrópolis (RJ): Vozes; 2007. p.297-322.

18.Spezani RS, Oliveira DC. O perfil da produção científica sobre identidade no campo da teoria das representações sociais. Psicol. teor. prat. [Internet]. 2013 [citado em 29 maio 2017]; 15(2): 10418. Disponível em: http://editorarevistas.mackenzie.br/index. $\mathrm{php} / \mathrm{ptp} / \mathrm{article} / \mathrm{view} / 4149 / 4454$

19. Cruz EJER, Souza NVDO, Correa RA, Pires AS. Dialectic feelings of the intensive care nurse about the work in Intensive Care. Esc. Anna Nery Rev. Enferm. 2014 [ cited 2018 Jan 10 ]; 18(3): 479-85. DOI: http://dx.doi.org/10.5935/1414-8145.20140068

20. Rocha APF, Souza KR, Teixeira LR. A saúde e o trabalho de médicos de UTI neonatal: um estudo em hospital público no Rio de Janeiro. Physis. 2015 [ citado em 10 jan 2018 ]; 25(3): 843-62. DOI: http://dx.doi.org/10.1590/S0103-73312015000300009.

21. Nascimento KC, Tosoli AM, Erdmann AL. Representational structure of intensive care for professionals working in mobile intensive care units. Rev. Esc. Enferm. USP. 2013 [ cited 2018 Jan 10 ]; 47(1):176-84. DOI: http://dx.doi.org/10.1590/S008062342013000100022

22. Ribeiro GSR, Silva RC, Ferreira MA. Technologies in intensive care: causes of adverse events and implications to nursing. Rev. bras. enferm. (Online). 2016 [ cited 2018 Jan 10 ]; 69(5): 972-80. DOI: http://dx.doi.org/10.1590/0034-7167.2016690505.

23. Bourgain JL, Coisel Y, Kern D, Nouette-Gaulain K, PanczerM. What are the main "machine dysfunctions" to know? Ann. Fr. Anesth. Reanim. 2014 [ cited 2018 Jan 10 ]; 33(7-8): 466-71. DOI: http://dx.doi.org/doi: 10.1016/j.annfar.2014.07.744.

24. Ribeiro GSR, Silva RC, Ferreira MA, Silva GR, Campos JF, Andrade BRP. Equipment failure:

25. conducts of nurses and implications for patient safety. Rev. bras. enferm. (Online). 2018 [ cited 2018 Sep 10 ]; 71(4): 1832-40. DOI: http://dx.doi.org/10.1590/0034-7167-2016-0547

26. Medeiros AC, Siqueira HCH, Zamberlan C, Cecagno D, Nunes SS, Thurow MRB. Comprehensiveness and humanization of nursing care management in the Intensive Care Unit. Esc. Enferm. USP. 2016 [ cited 2018 Jan 10 ]; 50(5): 816-22. DOI: http://dx.doi. org/10.1590/S0080-623420160000600015

27. Michelan VCA, Spiri WC. Perception of nursing workers humanization under intensive therapy. Rev. bras. enferm. (Online). 2018 [cited 2018 Jn 11 ] ; 71(2): 372-8. DOI: http://dx.doi. org/10.1590/0034-7167-2016-0485 